\title{
Estimates of Intrinsic Square Functions on Generalized Weighted Morrey Spaces
}

\author{
Guilian Gao' and Xiaomei $\mathrm{Wu}^{2}$ \\ ${ }^{1}$ School of Science, Hangzhou Dianzi University, Hangzhou 310018, China \\ ${ }^{2}$ Department of Mathematics, Zhejiang Normal University, Jinhua 321004, China
}

Correspondence should be addressed to Xiaomei Wu; wuxm@zjnu.cn

Received 24 March 2014; Accepted 8 July 2014; Published 22 July 2014

Academic Editor: Aurelian Gheondea

Copyright (C) 2014 G. Gao and X. Wu. This is an open access article distributed under the Creative Commons Attribution License, which permits unrestricted use, distribution, and reproduction in any medium, provided the original work is properly cited.

We prove the boundedness of the intrinsic functions on generalized weighted Morrey spaces $M^{p, \varphi}(w)$, including the strong type estimates and weak type estimates. Moreover, we define the $k$ th-order commutators generated by BMO $\left(\mathbb{R}^{n}\right)$ functions and intrinsic functions, and obtain their strong type estimates on $M^{p, \varphi}(w)$. In some cases, we improve previous results.

\section{Introduction}

It is well known that the theory of Littlewood-Paley square functions plays an important role in harmonic analysis, such as in the study of Fourier multiplier and singular integral operators. About their detailed properties and applications, we refer the readers to [1-3].

For convenience, let us recall some definitions. Suppose $u(x, t)=\left(P_{t} * f\right)(x)$ is the Poisson integral of $f$, where $P_{t}(x)$ denotes the Poisson kernel in $\mathbb{R}_{+}^{n+1}=\mathbb{R}^{n} \times(0, \infty)$. The Littlewood-Paley $g$-function and the Lusin area integral (square function) $S_{\beta}$ are defined, respectively, by

$$
\begin{gathered}
g(f)(x)=\left(\int_{0}^{\infty}|\nabla u(x, t)|^{2} t d t\right)^{1 / 2}, \\
S_{\beta}(f)(x)=\left(\iint_{\Gamma_{\beta}(x)}|\nabla u(y, t)|^{2} \frac{d y d t}{t^{n-1}}\right)^{1 / 2},
\end{gathered}
$$

where $\Gamma_{\beta}(x)=\left\{(y, t) \in \mathbb{R}_{+}^{n+1}:|x-y|<\beta t\right\}$ for any $\beta>0$. If $\beta=1$, set $S_{\beta}(f)=S(f)$. The corresponding $g_{\lambda}^{*}$-function $g_{\lambda}^{*}(f)$ is given by

$$
g_{\lambda}^{*}(f)(x)=\left(\iint_{\mathbb{R}_{+}^{n+1}}\left(\frac{t}{t+|x-y|}\right)^{n \lambda}|\nabla u(y, t)|^{2} \frac{d y d t}{t^{n-1}}\right)^{1 / 2} .
$$

Let $\psi \in C_{c}^{\infty}\left(\mathbb{R}^{n}\right)$ be real and radial and have support contained in $\left\{x \in \mathbb{R}^{n}:|x| \leq 1\right\}, \int_{\mathbb{R}^{n}} \phi(x) d x=0$. The continuous square functions $g_{\psi}(f)$ and $S_{\psi, \beta}(f)$ are also defined by

$$
\begin{gathered}
g_{\psi}(f)(x)=\left(\int_{0}^{\infty}\left|\left(\psi_{t} * f\right)(x)\right|^{2} \frac{d t}{t}\right)^{1 / 2}, \\
S_{\psi, \beta}(f)(x)=\left(\iint_{\Gamma_{\beta}(x)}\left|\left(\psi_{t} * f\right)(y)\right|^{2} \frac{d y d t}{t^{n+1}}\right)^{1 / 2},
\end{gathered}
$$

where $\psi_{t}$ denotes the usual $L^{1}$ dilation of $\psi: \psi_{t}(y)=t^{-n} \psi$ $(y / t)$.

Recently, Wilson [4] introduced a natural substitute for the above square functions, which he called the intrinsic square function. This function dominates pointwise all the above square functions and is independent of any particular kernel. At the same time, it is not essentially larger than any particular $S_{\psi, \beta}(f)$. For $0<\alpha \leq 1$, let $\mathscr{C}_{\alpha}$ be the family of functions $\phi$ having their support in $\left\{x \in \mathbb{R}^{n}:|x| \leq 1\right\}$, $\int_{\mathbb{R}^{n}} \phi(x) d x=0$, and for all $x$ and $y,|\phi(x)-\phi(y)| \leq|x-y|^{\alpha}$. If $f \in L_{\text {loc }}^{1}\left(\mathbb{R}^{n}\right)$ and $(y, t) \in \mathbb{R}_{+}^{n+1}$, we define

$$
A_{\alpha}(f)(y, t) \equiv \sup _{\phi \in \mathscr{C}_{\alpha}}\left|\left(\phi_{t} * f\right)(y)\right|
$$


where $\phi_{t}(y)=t^{-n} \phi(y / t)$. Then the intrinsic square of $f$ (of order $\alpha$ ) is defined by

$$
G_{\alpha, \beta}(f)(x)=\left(\iint_{\Gamma_{\beta}(x)}\left(A_{\alpha}(f)(y, t)\right)^{2} \frac{d y d t}{t^{n+1}}\right)^{1 / 2}
$$

Here and below, we drop the subscript $\beta$ if $\beta=1$. Although the function $G_{\alpha, \beta}(f)$ is depend of kernels with uniform compact support, there is pointwise relation between $G_{\alpha, \beta}(f)$ with different $\beta \geq 1$ :

$$
G_{\alpha, \beta}(f)(x) \leq \beta^{3 n / 2+\alpha} G_{\alpha}(f)(x), \quad x \in \mathbb{R}^{n} .
$$

See [4] for more details. The intrinsic Littlewood-Paley $g$ function and the intrinsic $g_{\lambda}^{*}$-function are defined by

$$
\begin{gathered}
g_{\alpha}(f)(x)=\left(\int_{0}^{\infty}\left(A_{\alpha}(f)(y, t)\right)^{2} \frac{d t}{t}\right)^{1 / 2}, \\
g_{\lambda, \alpha}^{*}(f)(x)=\left(\iint_{\mathbb{R}_{+}^{n+1}}\left(\frac{t}{t+|x-y|}\right)^{n \lambda}\right. \\
\left.\quad \times\left(A_{\alpha}(f)(y, t)\right)^{2} \frac{d y d t}{t^{n+1}}\right)^{1 / 2},
\end{gathered}
$$

respectively. In $[4,5]$, Wilson established the boundedness of intrinsic square functions on weighted Lesbesgue spaces. Their boundedness on various function spaces and their sharp bounds have received great attentions; see [6-17].

The classical Morrey spaces $L^{p, \lambda}\left(\mathbb{R}^{n}\right)$ were introduced by Morrey [18] to study the local behavior of solutions to secondorder elliptic partial differential equations. Mizuhara [19] introduced generalized Morrey spaces. Later, Guliyev defined the generalized Morrey spaces $M^{p, \varphi}\left(\mathbb{R}^{n}\right)$ with normalized norm. Recently, Komori and Shirai [20] first defined the weighted Morrey spaces $L^{p, \kappa}(w)$ and studied the boundedness of some classical operators on these spaces. Guliyev et al. $[21,22]$ defined the generalized weighted Morrey spaces $M^{p, \varphi}(w)$ as follows.

Definition 1. Let $\varphi(x, r)$ be a positive measurable function on $\mathbb{R}^{n} \times \mathbb{R}^{+}$and let $w(x)$ be a weight function on $\mathbb{R}^{n}$. The generalized weighted Morrey space $M^{p, \varphi}(w)$ is the space of all functions $f \in L_{\text {loc }}^{p, w}\left(\mathbb{R}^{n}\right)$ with finite norm

$$
\begin{aligned}
\|f\|_{M^{p, \varphi}(w)}= & \sup _{\substack{x \in \mathbb{R}^{n} \\
r>0}} \frac{1}{\varphi(x, r) w(B(x, r))^{1 / p}} \\
& \times\left(\int_{B(x, r)}|f(y)|^{p} w(y) d y\right)^{1 / p},
\end{aligned}
$$

where $w(B(x, r))=\int_{B(x, r)} w(y) d y$. The weak generalized weighted Morrey space $W M^{p, \varphi}(w)$ consists of all functions $f \in W L_{\text {loc }}^{p, w}\left(\mathbb{R}^{n}\right)$ with finite norm

$$
\|f\|_{W M^{p, \varphi}(w)}=\sup _{\substack{x \in \mathbb{R}^{n} \\ r>0}} \frac{1}{\varphi(x, r) w(B(x, r))^{1 / p}}\|f\|_{W L_{w}^{p}(B(x, r))},
$$

where

$$
\|f\|_{W L_{w}^{p}(B(x, r))}=\sup _{t>0}\left(\int_{\{y \in B(x, r):|f(y)|>t\}} w(y) d y\right)^{1 / p} .
$$

If $w(x) \equiv 1$ and $\varphi(x, r)=r^{(\lambda-n) / p}$ with $0<\lambda<n$, then $M^{p, \varphi}(w)=L^{p, \lambda}\left(\mathbb{R}^{n}\right)$; if $w(x) \equiv 1$, then $M^{p, \varphi}(1)=M^{p, \varphi}\left(\mathbb{R}^{n}\right)$; if $\varphi(x, r)=w(B(x, r))^{(\kappa-1) / p}$ for $0<\kappa<1$, then $M^{p, \varphi}(w)=$ $L^{p, \kappa}(w)$. There are many papers that discussed the conditions on $\varphi(x, r)$ to obtain the boundedness of operators on the generalized Morrey spaces $M^{p, \varphi}\left(\mathbb{R}^{n}\right)$. For example, see $[6,23-$ 29]. Recently, Guliyev introduced the generalized conditions:

$$
\begin{aligned}
& \int_{r}^{\infty} \frac{\operatorname{ess~inf}_{t<s<\infty} \varphi_{1}(x, s) w(B(x, s))^{1 / p}}{w(B(x, t))^{1 / p}} \frac{d t}{t} \leq C \varphi_{2}(x, r), \\
& \int_{r}^{\infty} \ln ^{k}\left(e+\frac{t}{r}\right) \frac{\operatorname{ess} \inf _{t<s<\infty} \varphi_{1}(x, s) w(B(x, s))^{1 / p}}{w(B(x, t))^{1 / p}} \frac{d t}{t} \\
& \quad \leq C \varphi_{2}(x, r),
\end{aligned}
$$

where $C$ does not depend on $x$ and $r$. Under these conditions, the boundedness of some classical operators and commutators in generalized weighted Morrey spaces were obtained, respectively. See $[8,9,21,22,30]$.

Recently, Wang $[12,16]$ studied the boundedness of the intrinsic functions and their commutators on weighted Morrey spaces. In $[6,17]$, the authors obtained the boundedness of the intrinsic functions and their commutators on generalized Morrey spaces. Guliyev et al. [7-9] obtained the estimates for vector-valued intrinsic square functions and their $k$ thorder commutators on vector-valued generalized weighted Morrey spaces. In this paper, we prove the boundedness of the intrinsic functions and their $k$ th-order commutators on generalized weighted Morrey spaces $M^{p, \varphi}(w)$ under the conditions (11), (12), and $w \in A_{p}$, respectively. Our partial results coincide with some results [7-9], but we improve known results in some case. See Remark 7.

The rest of the paper is organized as follows: some definitions and our main results are stated in Section 2. In Section 3, we give some lemmas. Finally, in Section 4, we prove our main theorem. Throughout this paper, $A \preceq B$ means that there is a positive constant $C$ independent of all essential variables such that $A \leq C B$. If $A \preceq B$ and $B \preceq A$, then we write $A \approx B$. We denote the conjugate exponent of $p>1$ by $p^{\prime}=p /(p-1)$ and $p^{\prime}=\infty$ if $p=1$.

\section{Definitions and Main Results}

In this paper, $B=B\left(x_{0}, r\right)$ denotes the ball with center $x_{0}$ and radius $r$. Given a ball $B$ and $\lambda>0, \lambda B$ denotes the ball with the same center as $B$ whose radius is $\lambda$ times that of $B$, and $B^{c}=\mathbb{R}^{n} \backslash B$. A weight is a locally integrable function on $\mathbb{R}^{n}$ which takes values in $(0, \infty)$ almost everywhere. For a weight $w$ and a measurable set $E$, we denote the characteristic function of $E$ by $\chi_{E}$, the Lebesgue measure of $E$ by $|E|$ and the 
weighted measure of $E$ by $w(E)$, where $w(E)=\int_{E} w(x) d x$. Moreover, for a locally integrable function $b(x)$, we define

$$
b_{B(x, r), w}=\frac{1}{w(B(x, r))} \int_{B(x, r)} b(y) w(y) d y .
$$

For simplicity, we denote $b_{B(x, r), w}$ by $b_{B(x, r)}$ if $w=1$.

Definition 2 (see [1]). A locally integrable function $b(x)$ is in $\operatorname{BMO}\left(\mathbb{R}^{n}\right)$ if

$$
\|b\|_{*}=\sup _{\substack{x \in \mathbb{R}^{n} \\ r>0}} \frac{1}{|B(x, r)|} \int_{B(x, r)}\left|b(y)-b_{B(x, r)}\right| d y<\infty .
$$

Definition 3 (see [3]). We say that a weight function $w \in A_{p}$, $1<p<\infty$, if

$$
\begin{aligned}
{[w]_{A_{p}}=} & \sup _{B}\left(\frac{1}{|B|} \int_{B} w(x) d x\right) \\
& \times\left(\frac{1}{|B|} \int_{B} w(x)^{-1 /(p-1)} d x\right)^{p-1}<\infty,
\end{aligned}
$$

where the supremum is taken over all balls $B$ in $\mathbb{R}^{n}$. A weight function $w \in A_{\infty}$ if $w \in A_{p}$ for some $1<p<\infty$. A weight function $w \in A_{1}$, if

$$
\frac{1}{|B|} \int_{B} w(x) d x \leq C \cdot \underset{x \in B}{\operatorname{ess} \inf } w(x) .
$$

Definition 4 . Let $b$ be a locally integrable function on $\mathbb{R}^{n}$ and

$$
\begin{aligned}
& A_{\alpha, b}^{k}(f)(y, t) \\
& \quad \equiv \sup _{\phi \in \mathscr{C}_{\alpha}}\left|\int_{\mathbb{R}^{n}}[b(x)-b(z)]^{k} \phi_{t}(y-z) f(z) d z\right| .
\end{aligned}
$$

The $k$ th-order commutators $\left[b, G_{\alpha}\right]^{k}$ and $\left[b, g_{\alpha}\right]^{k}$ are defined by

$$
\begin{gathered}
{\left[b, G_{\alpha}\right]^{k}(f)(x)=\left(\iint_{\Gamma(x)}\left(A_{\alpha, b}^{k}(f)(y, t)\right)^{2} \frac{d y d t}{t^{n+1}}\right)^{1 / 2},} \\
{\left[b, g_{\alpha}\right]^{k}(f)(x)=\left(\int_{0}^{\infty}\left(A_{\alpha, b}^{k}(f)(y, t)\right)^{2} \frac{d t}{t}\right)^{1 / 2},}
\end{gathered}
$$

respectively. Analogously, the $k$ th-order commutators [ $b$, $\left.g_{\lambda, \alpha}^{*}\right]^{k}$ are defined by

$$
\begin{aligned}
{\left[b, g_{\lambda, \alpha}^{*}\right]^{k}(f)(x)=\left(\iint_{\mathbb{R}_{+}^{n+1}}\right.} & \left(\frac{t}{t+|x-y|}\right)^{\lambda n} \\
& \left.\times\left(A_{\alpha, b}^{k}(f)(y, t)\right)^{2} \frac{d y d t}{t^{n+1}}\right)^{1 / 2} .
\end{aligned}
$$

Theorem 5. Let $0<\alpha \leq 1,1 \leq p<\infty, w \in A_{p}$ and $\left(\varphi_{1}, \varphi_{2}\right)$ satisfy condition (11). Then,

$$
\begin{gathered}
\left\|G_{\alpha}(f)\right\|_{M^{p, \varphi_{2}}(w)} \preceq\|f\|_{M^{p, \varphi_{1}}(w)}, \quad 1<p<\infty, \\
\left\|G_{\alpha}(f)\right\|_{W M^{1, \varphi_{2}}(w)} \preceq\|f\|_{M^{1, \varphi_{1}}(w)}, \quad p=1 .
\end{gathered}
$$

Theorem 6. Let $0<\alpha \leq 1,1 \leq p<\infty, w \in A_{p}$, and $\left(\varphi_{1}, \varphi_{2}\right)$ satisfy condition (11). Then for $\lambda>3+2 \alpha / n$, we have

$$
\begin{gathered}
\left\|g_{\lambda, \alpha}^{*}(f)\right\|_{M^{p, \varphi_{2}}(w)} \preceq\|f\|_{M^{p, \varphi_{1}}(w)}, \quad 1<p<\infty, \\
\left\|g_{\lambda, \alpha}^{*}(f)\right\|_{W M^{1, \varphi_{2}}(w)} \preceq\|f\|_{M^{1, \varphi_{1}}(w)}, \quad p=1 .
\end{gathered}
$$

Theorem $6^{\prime}$. Let $0<\alpha \leq 1,1<p<\infty, w \in A_{p}$ and let $\left(\varphi_{1}, \varphi_{2}\right)$ satisfy condition (11). Then, for $\lambda>\max \{p, 3\}$, we have

$$
\left\|g_{\lambda, \alpha}^{*}(f)\right\|_{M^{p, \varphi_{2}}(w)} \preceq\|f\|_{M^{p, \varphi_{1}}(w)} .
$$

Remark 7. If $p>3+2 \alpha / n$, comparing Theorem 6 with Theorem $6^{\prime}$, we obtain that the conclusion of Theorem 6 is better. So in this sense, our Theorem 6 improves the result in [12]. If $1<p \leq 3$, the conclusion of Theorem $6^{\prime}$ is better than that of Theorem 6 . Therefore, we improve the corresponding result in $[6,17]$. Here, we point out that Theorem $6^{\prime}$ and Theorem $9^{\prime}$ can be extended to vector-valued cases; see [79].

Theorem 8. Let $0<\alpha \leq 1,1<p<\infty, w \in A_{p}$, and $\left(\varphi_{1}, \varphi_{2}\right)$ satisfy condition (12). Let $b \in B M O\left(\mathbb{R}^{n}\right)$; then

$$
\left\|\left[b, G_{\alpha}\right]^{k}(f)\right\|_{M^{p, \varphi_{2}}(w)} \preceq\|b\|_{*}^{k}\|f\|_{M^{p, \varphi}\left(w_{1}\right)} .
$$

Theorem 9. Let $0<\alpha \leq 1,1<p<\infty, w \in A_{p}$, and $\left(\varphi_{1}, \varphi_{2}\right)$ satisfy condition (12). If $b \in B M O\left(\mathbb{R}^{n}\right)$ and $\lambda>3+2 \alpha / n$, then

$$
\left\|\left[b, g_{\lambda, \alpha}^{*}\right]^{k}(f)\right\|_{M^{p, \varphi_{2}}(w)} \preceq\|b\|_{*}^{k}\|f\|_{M^{p, \varphi_{1}}(w)} .
$$

Theorem $9^{\prime}$. Let $0<\alpha \leq 1,1<p<\infty, w \in A_{p}$, and $\left(\varphi_{1}, \varphi_{2}\right)$ satisfy condition (12). If $b \in B M O\left(\mathbb{R}^{n}\right)$ and $\lambda>\max \{p, 3\}$, then

$$
\left\|\left[b, g_{\lambda, \alpha}^{*}\right]^{k}(f)\right\|_{M^{p, \varphi_{2}}(w)} \preceq\|b\|_{*}^{k}\|f\|_{M^{p, \varphi_{1}}(w)} .
$$

For $0<\alpha \leq 1$, Wilson [4] showed that $G_{\alpha} f$ and $g_{\alpha} f$ are pointwise comparable. Therefore, by Theorems 5 and 8 , we have the following.

Corollary 10. Let $0<\alpha \leq 1,1 \leq p<\infty, w \in A_{p}$, and $\left(\varphi_{1}, \varphi_{2}\right)$ satisfy condition (11); then

$$
\begin{gathered}
\left\|g_{\alpha}(f)\right\|_{M^{p, \varphi_{2}}(w)} \preceq\|f\|_{M^{p, \varphi_{1}}(w)}, \quad 1<p<\infty, \\
\left\|g_{\alpha}(f)\right\|_{W M^{1, \varphi_{2}}(w)} \preceq\|f\|_{M^{1, \varphi_{1}}(w)}, \quad p=1 .
\end{gathered}
$$


Corollary 11. Let $0<\alpha \leq 1,1<p<\infty, w \in A_{p}$, and $\left(\varphi_{1}, \varphi_{2}\right)$ satisfy condition (12). If $b \in B M O\left(\mathbb{R}^{n}\right)$, then

$$
\left\|\left[b, g_{\alpha}\right]^{k}(f)\right\|_{M^{p, \varphi_{2}}(w)} \leq\|b\|_{*}^{k}\|f\|_{M^{p, \varphi_{1}}(w)} .
$$

Remark 12. If $\varphi(x, r)=w(B(x, r))^{(\kappa-1) / p}$ for $0<\kappa<1$, then $M^{p, \varphi}(w)=L^{p, \kappa}(w)$. Let $w \in A_{p}(1 \leq p<\infty)$; the pair $\left(\varphi_{1}, \varphi_{2}\right)$ satisfies the condition (12) with $k \geq 0$. See [30] for its proof. Therefore, Theorems 5, 6', 8, $9^{\prime}$ and Corollaries 10 and 11 contain the results in $[12,16]$.

Remark 13. If $1<p<\infty, w=1$, and $k=1$, then Theorems 5-9 and Corollaries 10 and 11 contain the results in $[6,17]$.

\section{Lemmas}

For any $x \in \mathbb{R}^{n}$ and $\beta \geq 1$, the following inequality proved in [4] holds:

$$
G_{\alpha, \beta}(f)(x) \leq \beta^{3 n / 2+\alpha} G_{\alpha}(f)(x) .
$$

So we can readily obtain the following.

Lemma 14. Let $0<\alpha \leq 1$ and $1<p<\infty$. If $w$ is a weight, we have

$$
\left\|G_{\alpha, 2^{j}}(f)\right\|_{L_{w}^{p}\left(\mathbb{R}^{n}\right)} \leq 2^{j(3 n / 2+\alpha)}\left\|G_{\alpha}(f)\right\|_{L_{w}^{p}\left(\mathbb{R}^{n}\right)}, \quad \forall j \in \mathbb{Z}^{+} .
$$
ing.

By the similar argument as in [12], we can get the follow-

Lemma 15. Let $0<\alpha \leq 1,1<p<\infty$, and $w \in A_{p}$. Then, the $k$ th-order commutators $\left[b, G_{\alpha}\right]^{k}$ and $\left[b, g_{\lambda, \alpha}^{*}\right]^{k}$ are all bounded from $L_{w}^{p}\left(\mathbb{R}^{n}\right)$ to itself whenever $b \in B M O\left(\mathbb{R}^{n}\right)$.

In the following, we will give a lemma about the Hardy type operator:

$$
H_{k}(f)(r):=\frac{1}{r} \int_{0}^{r} \ln ^{k}\left(e+\frac{r}{t}\right) f(t) d \mu(t), \quad k \geq 0,
$$

where $\mu$ is a nonnegative Borel measure on $(0, \infty)$; we have the following.

Lemma 16 (see [30]). The inequality

$$
\underset{r>0}{\operatorname{esssup}} H_{k}(f)(r) W(r) \leq C \underset{r>0}{\operatorname{esssup}} f(r) V(r)
$$

holds for all nonnegative and nonincreasing $f$ on $(0, \infty)$ if and only if

$$
A:=\sup _{r>0} \frac{W(r)}{r} \int_{0}^{r} \ln ^{k}\left(e+\frac{r}{t}\right) \frac{d \mu(t)}{\operatorname{ess} \sup _{0<s<t} V(s)}<\infty,
$$

and $C \approx A$.

Note that if $k=0$ and $d \mu(t)=d t$, Lemma 16 was proved in [31].
Lemma 17 (see [30]). Let $w \in A_{\infty}$ and $b \in B M O\left(\mathbb{R}^{n}\right)$. Suppose $k>0$ and $r_{1}, r_{2}>0$. Then,

(i) for $1 \leq p<\infty$, we have

$$
\begin{aligned}
& \left(\frac{1}{w\left(B\left(x, r_{1}\right)\right)} \int_{B\left(x, r_{1}\right)}\left|b(y)-b_{B\left(x, r_{2}\right), w}\right|^{k p} w(y) d y\right)^{1 / p} \\
& \quad \leq C\left(1+\left|\ln \frac{r_{1}}{r_{2}}\right|\right)^{k}\|b\|_{*}^{k},
\end{aligned}
$$

where $C$ is independent of $b, w, x, r_{1}$, and $r_{2}$.

(ii) for $1<p<\infty$, we have

$$
\begin{aligned}
& \left(\frac{1}{w^{1-p^{\prime}}\left(B\left(x, r_{1}\right)\right)}\right. \\
& \left.\quad \times \int_{B\left(x, r_{1}\right)}\left|b(y)-b_{B\left(x, r_{2}\right), w}\right|^{k p^{\prime}} w(y)^{1-p^{\prime}} d y\right)^{1 / p^{\prime}} \\
& \leq C\left(1+\left|\ln \frac{r_{1}}{r_{2}}\right|\right)^{k}\|b\|_{*}^{k},
\end{aligned}
$$

where $C$ is independent of $b, w, x, r_{1}$, and $r_{2}$.

Lemma 18 (see [12]). Let $0<\alpha \leq 1,1<p<\infty$, and $w \in A_{p}$. Then, for any $j \in \mathbb{Z}^{+}$, we have

$$
\left\|G_{\alpha, 2^{j}}(f)\right\|_{L_{w}^{p}\left(\mathbb{R}^{n}\right)} \leq \begin{cases}2^{j n}\left\|G_{\alpha}(f)\right\|_{L_{w}^{p}\left(\mathbb{R}^{n}\right)}, & 1 \leq p \leq 2 ; \\ 2^{j n p / 2}\left\|G_{\alpha}(f)\right\|_{L_{w}^{p}\left(\mathbb{R}^{n}\right)}, & 2<p<\infty .\end{cases}
$$

\section{Proofs of Main Theorems}

Proof of Theorem 5. We will adopt the idea used in [27]. Fix a ball $B=B\left(x_{0}, r\right)$ and decompose $f=f_{1}+f_{2}$, where $f_{1}=f \chi_{2 B}$ and $f_{2}=f \chi_{(2 B)}$. Then, for $1<p<\infty$, we have

$$
\left\|G_{\alpha}(f)\right\|_{L_{w}^{p}(B)} \leq\left\|G_{\alpha}\left(f_{1}\right)\right\|_{L_{w}^{p}(B)}+\left\|G_{\alpha}\left(f_{2}\right)\right\|_{L_{w}^{p}(B)} .
$$

Since $w \in A_{p}$ for $1<p<\infty$, we can obtain the following inequality from [5]

$$
\left\|G_{\alpha}\left(f_{1}\right)\right\|_{L_{w}^{p}(B)} \preceq\left\|f_{1}\right\|_{L_{w}^{p}\left(\mathbb{R}^{n}\right)}=\|f\|_{L_{w}^{p}(2 B)} .
$$

For $\|f\|_{L_{w}^{p}(2 B)}$ and $w \in A_{p}$ with $1 \leq p<\infty$, a calculation shows that

$$
\|f\|_{L_{w}^{p}(2 B)} \leq[w]_{A_{p}}^{1 / p} w(B)^{1 / p} \int_{2 r}^{\infty} \frac{\|f\|_{L_{w}^{p}\left(B\left(x_{0}, t\right)\right)}}{w\left(B\left(x_{0}, t\right)\right)^{1 / p}} \frac{d t}{t} .
$$

See [30] for its proof. Therefore, for $1<p<\infty$, we get

$$
\left\|G_{\alpha}\left(f_{1}\right)\right\|_{L_{w}^{p}(B)} \leq[w]_{A_{p}}^{1 / p} w(B)^{1 / p} \int_{2 r}^{\infty} \frac{\|f\|_{L_{w}^{p}\left(B\left(x_{0}, t\right)\right)}}{w\left(B\left(x_{0}, t\right)\right)^{1 / p}} \frac{d t}{t} .
$$


On the other hand, for $\phi \in \mathscr{C}_{\alpha}$ with $0<\alpha \leq 1$, we have

$$
\left|f_{2} * \phi_{t}(y)\right| \preceq t^{-n} \int_{|y-z| \leq t}\left|f_{2}(z)\right| d z .
$$

Since $x \in B, z \in(2 B)^{c}$, and $(y, t) \in \Gamma(x)$, we have $r \leq|z-x| \leq$ $2 t$. So we obtain

$$
\begin{aligned}
G_{\alpha}\left(f_{2}\right)(x) & \leq\left(\iint_{\Gamma(x)}\left|t^{-n} \int_{|y-z| \leq t}\right| f_{2}(z)|d z|^{2} \frac{d y d t}{t^{n+1}}\right)^{1 / 2} \\
& \leq\left(\int_{t \geq r / 2} \int_{|x-y|<t}\left(\int_{|z-x| \leq 2 t}\left|f_{2}(z)\right| d z\right)^{2} \frac{d y d t}{t^{3 n+1}}\right)^{1 / 2} \\
& \preceq\left(\int_{t \geq \frac{r}{2}}\left(\int_{|z-x| \leq 2 t}\left|f_{2}(z)\right| d z\right)^{2} \frac{d t}{t^{2 n+1}}\right)^{1 / 2} .
\end{aligned}
$$

By Minkowski's inequality and $|z-x| \geq(1 / 2)\left|z-x_{0}\right|$ for $x \in B$ and $z \in(2 B)^{c}$, we have

$$
\begin{aligned}
G_{\alpha}\left(f_{2}\right)(x) & \preceq \int_{\mathbb{R}^{n}}\left(\int_{t>|z-x| / 2} \frac{d t}{t^{2 n+1}}\right)^{1 / 2}\left|f_{2}(z)\right| d z \\
& \preceq \int_{\left|z-x_{0}\right| \geq 2 r} \frac{|f(z)| d z}{|z-x|^{n}} \preceq \int_{(2 B)^{c}} \frac{|f(z)| d z}{\left|z-x_{0}\right|^{n}} \\
& \approx \int_{(2 B)^{c}}|f(z)| \int_{\left|z-x_{0}\right|}^{\infty} \frac{d t d z}{t^{n+1}} .
\end{aligned}
$$

It follows from Fubini's theorem, Hölder's inequality, and $w \in$ $A_{p}$ with $1 \leq p<\infty$ that

$$
\begin{aligned}
G_{\alpha}\left(f_{2}\right)(x) & \preceq \int_{2 r}^{\infty} \int_{B\left(x_{0}, t\right)} \frac{|f(z)| d z d t}{t^{n+1}} \\
& \preceq \int_{2 r}^{\infty}\|f\|_{L_{w}^{p}\left(B\left(x_{0}, t\right)\right)}\left\|w^{-1 / p}\right\|_{L^{p^{\prime}}\left(B\left(x_{0}, t\right)\right)} \frac{d t}{t^{n+1}} \\
& \preceq[w]_{A_{p}}^{1 / p} \int_{2 r}^{\infty} \frac{\|f\|_{L_{w}^{p}\left(B\left(x_{0}, t\right)\right)}}{w\left(B\left(x_{0}, t\right)\right)^{1 / p}} \frac{d t}{t} .
\end{aligned}
$$

Thus, for $1 \leq p<\infty$, the following estimate is valid:

$$
\left\|G_{\alpha}\left(f_{2}\right)\right\|_{L_{w}^{p}(B)} \preceq[w]_{A_{p}}^{1 / p} w(B)^{1 / p} \int_{2 r}^{\infty} \frac{\|f\|_{L_{w}^{p}\left(B\left(x_{0}, t\right)\right)}}{w\left(B\left(x_{0}, t\right)\right)^{1 / p}} \frac{d t}{t} .
$$

Combining the above estimates (36), (39), and (44), we have

$$
\left\|G_{\alpha}(f)\right\|_{L_{w}^{p}(B)} \preceq[w]_{A_{p}}^{1 / p} w(B)^{1 / p} \int_{2 r}^{\infty} \frac{\|f\|_{L_{w}^{p}\left(B\left(x_{0}, t\right)\right)}}{w\left(B\left(x_{0}, t\right)\right)^{1 / p}} \frac{d t}{t} .
$$

Hence, applying the definition of $M^{p, \varphi}(w)$ and substitution of variables, we have

$$
\begin{aligned}
& \left\|G_{\alpha}(f)\right\|_{M^{p, \varphi_{2}}(w)} \\
& \quad \preceq \sup _{\substack{x_{0} \in \mathbb{R}^{n} \\
r>0}} \varphi_{2}\left(x_{0}, r^{-1}\right)^{-1} \int_{0}^{r} \frac{\|f\|_{L_{w}^{p}\left(B\left(x_{0}, s^{-1}\right)\right)}}{w\left(B\left(x_{0}, s^{-1}\right)\right)^{1 / p}} \frac{d s}{s} .
\end{aligned}
$$

Suppose $W(r)=\varphi_{2}\left(x_{0}, r^{-1}\right)^{-1} r$ and $V(x)=\varphi_{1}\left(x_{0}, r^{-1}\right)^{-1}$ $w\left(B\left(x_{0}, r^{-1}\right)\right)^{-1 / p}$. Since $\left(\varphi_{1}, \varphi_{2}\right)$ satisfies condition (11), we can verify that $W(r), V(r)$ satisfy condition (32). Obviously, $\|f\|_{L_{w}^{p}\left(B\left(x_{0}, s^{-1}\right)\right)}$ is decreasing on variable $s$. So, by Lemma 16 with $k=0$, we can conclude

$$
\left\|G_{\alpha}(f)\right\|_{M^{p, \varphi_{2}}(w)} \preceq\|f\|_{M^{p, \varphi_{1}}(w)} .
$$

Let $p=1$. From $w \in A_{1}$, [5], (38) and (44), it follows that

$$
\begin{aligned}
\left\|G_{\alpha}(f)\right\|_{W L_{w}^{1}(B)} & \leq\left\|G_{\alpha}\left(f_{1}\right)\right\|_{W L_{w}^{1}(B)}+\left\|G_{\alpha}\left(f_{2}\right)\right\|_{W L_{w}^{1}(B)} \\
& \preceq\left\|f_{1}\right\|_{L_{w}^{1}\left(\mathbb{R}^{n}\right)}+\left\|G_{\alpha}\left(f_{2}\right)\right\|_{L_{w}^{1}(B)} \\
& \preceq w(B) \int_{2 r}^{\infty} \frac{\|f\|_{L_{w}^{1}\left(B\left(x_{0}, t\right)\right)}}{w\left(B\left(x_{0}, t\right)\right)} \frac{d t}{t} .
\end{aligned}
$$

By the definition of $W M_{1, \varphi}(w)$ and Lemma 16 with $k=0$, we get

$$
\begin{aligned}
& \left\|G_{\alpha}(f)\right\|_{W M^{1, \varphi_{2}}(w)} \\
& \quad \preceq \sup _{\substack{x_{0} \in \mathbb{R}^{n} \\
r>0}} r \varphi_{2}\left(x_{0}, r^{-1}\right)^{-1} \frac{1}{r} \int_{0}^{r} \frac{\|f\|_{L_{w}^{1}\left(B\left(x_{0}, s^{-1}\right)\right)} \frac{d s}{w\left(B\left(x_{0}, s^{-1}\right)\right)}}{s} \\
& \preceq\|f\|_{M^{1, \varphi_{1}}(w)} .
\end{aligned}
$$

Proof of Theorem 6. By the definition of $g_{\lambda, \alpha}^{*}(f)$, we have

$$
\begin{aligned}
& {\left[g_{\lambda, \alpha}^{*}(f)(x)\right]^{2}=} \int_{0}^{\infty} \int_{|x-y|<t}\left(\frac{t}{t+|x-y|}\right)^{n \lambda} \\
& \times\left(A_{\alpha} f(y, t)\right)^{2} \frac{d y d t}{t^{n+1}} \\
&+\sum_{j=1}^{\infty} \int_{0}^{\infty} \int_{2^{j-1} t \leq|x-y|<2^{j} t}\left(\frac{t}{t+|x-y|}\right)^{n \lambda} \\
& \preceq\left(G_{\alpha} f(x)\right)^{2}+\sum_{j=1}^{\infty} 2^{-j n \lambda}\left(G_{\alpha, 2^{j}}(f)(x)\right)^{2} .
\end{aligned}
$$

Thus,

$$
\begin{aligned}
\left\|g_{\lambda, \alpha}^{*}(f)\right\|_{M^{p, \varphi_{2}}(w)} \preceq & \left\|G_{\alpha}(f)\right\|_{M^{p, \varphi_{2}}(w)} \\
& +\sum_{j=1}^{\infty} 2^{-j n \lambda / 2}\left\|G_{\alpha, 2^{j}}(f)\right\|_{M^{p, \varphi_{2}}(w)} .
\end{aligned}
$$

By Theorem 5, we have

$$
\left\|G_{\alpha}(f)\right\|_{M^{p, \varphi_{2}}(w)} \preceq\|f\|_{M^{p, \varphi_{1}}(w)} .
$$


It remains to estimate $\left\|G_{\alpha, 2^{j}}(f)\right\|_{M^{p, w_{2}}\left(\mathbb{R}^{n}\right)}$. To estimate it, we will divide $\left\|G_{\alpha, 2^{j}}(f)\right\|_{L_{w}^{p}(B)}$ into two terms. As before,

$$
\left\|G_{\alpha, 2^{j}}(f)\right\|_{L_{w}^{p}(B)} \leq\left\|G_{\alpha, 2^{j}}\left(f_{1}\right)\right\|_{L_{w}^{p}(B)}+\left\|G_{\alpha, 2^{j}}\left(f_{2}\right)\right\|_{L_{w}^{p}(B)},
$$

where $f=f_{1}+f_{2}$ and $f_{1}=f \chi_{2 B}$. For the first term, by Lemma 14, Theorem 5, and (38), we can easily deduce

$$
\begin{aligned}
\left\|G_{\alpha, 2^{j}}\left(f_{1}\right)\right\|_{L_{w}^{p}(B)} & \preceq 2^{j(3 n / 2+\alpha)}\left\|G_{\alpha}\left(f_{1}\right)\right\|_{L_{w}^{p}\left(\mathbb{R}^{n}\right)} \\
& \preceq 2^{j(3 n / 2+\alpha)}\|f\|_{L_{w}^{p}(2 B)} \\
& \preceq 2^{j(3 n / 2+\alpha)} w(B)^{1 / p} \int_{2 r}^{\infty} \frac{\|f\|_{L_{w}^{p}\left(B\left(x_{0}, t\right)\right)}}{w\left(B\left(x_{0}, t\right)\right)^{1 / p}} \frac{d t}{t} .
\end{aligned}
$$

Now, we estimate the second term. For $\phi \in \mathscr{C}_{\alpha}$ with $0<\alpha \leq$ 1 , we have

$$
\begin{aligned}
& G_{\alpha, 2^{j}}\left(f_{2}\right)(x) \\
& \quad \preceq\left(\int_{0}^{\infty} \int_{|x-y| \leq 2^{j} t}\left(\int_{|z-y| \leq t}\left|f_{2}(z)\right| d z\right)^{2} \frac{d y d t}{t^{3 n+1}}\right)^{1 / 2} .
\end{aligned}
$$

Notice that $|x-y| \leq 2^{j} t$ and $|z-y| \leq t$, so $|z-x| \leq 2^{j+1} t$. Thus,

$$
\begin{aligned}
& G_{\alpha, 2^{j}}\left(f_{2}\right)(x) \\
& \quad \leq\left(\int_{0}^{\infty} \int_{|x-y| \leq 2^{j} t}\left(\int_{|z-x| \leq 2^{j+1} t}\left|f_{2}(z)\right| d z\right)^{2} \frac{d y d t}{t^{3 n+1}}\right)^{1 / 2} \\
& \quad \leq\left(\int_{0}^{\infty}\left(\int_{|z-x| \leq 2^{j+1} t}\left|f_{2}(z)\right| d z\right)^{2} \frac{2^{j n} d t}{t^{2 n+1}}\right)^{1 / 2} \\
& \quad \leq 2^{j n / 2} \int_{\mathbb{R}^{n}}\left(\int_{t \geq|z-x| / 2^{j+1}}\left|f_{2}(z)\right|^{2} \frac{d t}{t^{2 n+1}}\right)^{1 / 2} d z \\
& \quad \leq 2^{3 j n / 2} \int_{\left|z-x_{0}\right|>2 r} \frac{|f(z)|}{|z-x|^{n}} d z .
\end{aligned}
$$

Because $|z-x| \geq(1 / 2)\left|z-x_{0}\right|$ for $x \in B=B\left(x_{0}, r\right)$ and $\left|z-x_{0}\right|>2 r$, by Fubini's theorem and Hölder's inequality,

$$
\begin{aligned}
G_{\alpha, 2^{j}}\left(f_{2}\right)(x) & \leq 2^{3 j n / 2} \int_{\left|z-x_{0}\right|>2 r} \frac{|f(z)| d z}{\left|z-x_{0}\right|^{n}} \\
& \approx 2^{3 j n / 2} \int_{\left|z-x_{0}\right|>2 r}|f(z)| \int_{\left|z-x_{0}\right|}^{\infty} \frac{d t d z}{t^{n+1}} \\
& \leq 2^{3 j n / 2} \int_{2 r}^{\infty} \int_{B\left(x_{0}, t\right)} \frac{|f(z)| d z d t}{t^{n+1}} \\
& \leq 2^{3 j n / 2}[w]_{A_{p}}^{1 / p} \int_{2 r}^{\infty} \frac{\|f\|_{L_{w}^{p}\left(B\left(x_{0}, t\right)\right)}}{w\left(B\left(x_{0}, t\right)\right)^{1 / p}} \frac{d t}{t} .
\end{aligned}
$$

So

$$
\left\|G_{\alpha, 2^{j}}\left(f_{2}\right)\right\|_{L_{w}^{p}(B)} \preceq 2^{3 j n / 2} w(B)^{1 / p} \int_{2 r}^{\infty} \frac{\|f\|_{L_{w}^{p}\left(B\left(x_{0}, t\right)\right)}}{w\left(B\left(x_{0}, t\right)\right)^{1 / p}} \frac{d t}{t} .
$$

Combining (53), (54), and (58), we have

$$
\left\|G_{\alpha, 2^{j}}(f)\right\|_{L_{w}^{p}(B)} \preceq 2^{j(3 n / 2+\alpha)} w(B)^{1 / p} \int_{2 r}^{\infty} \frac{\|f\|_{L_{w}^{p}\left(B\left(x_{0}, t\right)\right)}}{w\left(B\left(x_{0}, t\right)\right)^{1 / p}} \frac{d t}{t} .
$$

Thus, by changing of variables and Lemma 16 with $k=0$, we get

$$
\begin{aligned}
\left\|G_{\alpha, 2^{j}}(f)\right\|_{M^{p, \varphi_{2}}(w)} \preceq 2^{j(3 n / 2+\alpha)} \sup _{\substack{x_{0} \in \mathbb{R}^{n} \\
r>0}} \varphi_{2}\left(B\left(x_{0}, r\right)\right)^{-1} \\
\\
\quad \times \int_{0}^{r} \frac{\|f\|_{L_{w}^{p}\left(B\left(x_{0}, t^{-1}\right)\right)} \frac{d t}{w\left(B\left(x_{0}, t^{-1}\right)\right)^{1 / p}}}{\preceq} \\
\end{aligned}
$$

Therefore, using (51), (52), and (60), it follows that

$$
\begin{aligned}
\left\|g_{\lambda, \alpha}^{*}(f)\right\|_{M^{p, \varphi_{2}}(w)} & \preceq\|f\|_{M^{p, \varphi_{1}}(w)}\left(1+\sum_{j=1}^{\infty} 2^{j n(\alpha / n-\lambda / 2+3 / 2)}\right) \\
& \preceq\|f\|_{M^{p, \varphi_{1}}(w)}
\end{aligned}
$$

where the series are convergent since $\lambda>3+2 \alpha / n$.

Proof of Theorem $6^{\prime}$. By Lemma 18, using the arguments as the proofs of Theorem 6 , we can finish the proof. The details are omitted here.

Proof of Theorem 8. Fix a ball $B=B\left(x_{0}, r\right)$ and decompose $f=f_{1}+f_{2}$, where $f_{1}=f \chi_{2 B}$. Then,

$$
\begin{aligned}
\left\|\left[b, G_{\alpha}\right]^{k}(f)\right\|_{L_{w}^{p}(B)} \leq & \left\|\left[b, G_{\alpha}\right]^{k}\left(f_{1}\right)\right\|_{L_{w}^{p}(B)} \\
& +\left\|\left[b, G_{\alpha}\right]^{k}\left(f_{2}\right)\right\|_{L_{w}^{p}(B)}
\end{aligned}
$$

By Lemma 15 and (38), we have that

$$
\begin{aligned}
\left\|\left[b, G_{\alpha}\right]^{k}\left(f_{1}\right)\right\|_{L_{w}^{p}(B)} & \leq\|b\|_{*}^{k}\left\|f_{1}\right\|_{L_{w}^{p}\left(\mathbb{R}^{n}\right)} \preceq\|b\|_{*}^{k}\|f\|_{L_{w}^{p}(2 B)} \\
& \preceq b \|_{*}^{k}[w]_{A_{p}}^{1 / p} w(B)^{1 / p} \\
& \times \int_{2 r}^{\infty} \frac{\|f\|_{L_{w}^{p}\left(B\left(x_{0}, t\right)\right)}}{w\left(B\left(x_{0}, t\right)\right)^{1 / p}} \frac{d t}{t} .
\end{aligned}
$$


To estimate $\left\|\left[b, G_{\alpha}\right]^{k}\left(f_{2}\right)\right\|_{L_{w}^{p}(B)}$, we divide $\left[b, G_{\alpha}\right]^{k}\left(f_{2}\right)$ into two parts:

$$
\begin{aligned}
& {\left[b, G_{\alpha}\right]^{k}\left(f_{2}\right)(x)} \\
& \leq\left(\iint_{\Gamma(x)} \sup _{\phi \in \mathscr{C}_{\alpha}} \mid \int_{\mathbb{R}^{n}}\left[b(x)-b_{B, w}\right]^{k}\right. \\
& \left.\quad \times\left.\phi_{t}(y-z) f_{2}(z) d z\right|^{2} \frac{d y d t}{t^{n+1}}\right)^{1 / 2} \\
& +\left(\iint_{\Gamma(x)} \sup _{\phi \in \mathscr{C}_{\alpha}} \mid \int_{\mathbb{R}^{n}}\left[b_{B, w}-b(z)\right]^{k}\right. \\
& \left.\times\left.\phi_{t}(y-z) f_{2}(z) d z\right|^{2} \frac{d y d t}{t^{n+1}}\right)^{1 / 2}
\end{aligned}
$$$$
:=J+J
$$

First, $J=\left|b(x)-b_{B, w}\right|^{k} G_{\alpha}\left(f_{2}\right)(x)$. From the proof of Theorem 5, we know

$$
G_{\alpha}\left(f_{2}\right)(x) \preceq[w]_{A_{p}}^{1 / p} \int_{2 r}^{\infty} \frac{\|f\|_{L_{w}^{p}\left(B\left(x_{0}, t\right)\right)}}{w\left(B\left(x_{0}, t\right)\right)^{1 / p}} \frac{d t}{t} .
$$

Hence, using Lemma 17, we obtain

$$
\begin{aligned}
\|J\|_{L_{w}^{p}(B)} \leq & {[w]_{A_{p}}^{1 / p} \int_{2 r}^{\infty} \frac{\|f\|_{L_{w}^{p}\left(B\left(x_{0}, t\right)\right)}}{w\left(B\left(x_{0}, t\right)\right)^{1 / p}} \frac{d t}{t} } \\
& \times\left(\int_{B}\left|b(x)-b_{B, w}\right|^{k p} w(x) d x\right)^{1 / p} \\
\leq\|b\|_{*}^{k} w(B)^{1 / p} & \int_{2 r}^{\infty} \frac{\|f\|_{L_{w}^{p}\left(B\left(x_{0}, t\right)\right)}}{w\left(B\left(x_{0}, t\right)\right)^{1 / p}} \frac{d t}{t} .
\end{aligned}
$$

For $J J$, note that $\phi \in \mathscr{C}_{\alpha}$ with $0<\alpha \leq 1$ and $|x-z|<2 t$; thus, by Minkowski's inequality,

$$
\begin{aligned}
J J & \leq\left(\iint_{\Gamma(x)}\left|\int_{|x-z|<2 t}\right| b(z)-\left.\left.b_{B, w}\right|^{k}\left|f_{2}(z)\right| d z\right|^{2} \frac{d y d t}{t^{3 n+1}}\right)^{1 / 2} \\
& \leq\left(\int_{0}^{\infty}\left|\int_{|x-z|<2 t}\right| b(z)-\left.\left.b_{B, w}\right|^{k}\left|f_{2}(z)\right| d z\right|^{2} \frac{d t}{t^{2 n+1}}\right)^{1 / 2} \\
& \leq \int_{\left|z-x_{0}\right|>2 r}\left|b(z)-b_{B, w}\right|^{k} \frac{|f(z)| d z}{|x-z|^{n}} \\
& \leq \int_{\left|z-x_{0}\right|>2 r}\left|b(z)-b_{B, w}\right|^{k} \frac{|f(z)| d z}{\left|z-x_{0}\right|^{n}} \\
& \approx \int_{\left|z-x_{0}\right|>2 r}\left|b(z)-b_{B, w}\right|^{k} \int_{\left|z-x_{0}\right|}^{\infty} \frac{|f(z)| d t d z}{t^{n+1}} .
\end{aligned}
$$

Applying Fubini's theorem, Hölder's inequality, and Lemma 17, we have

$$
\begin{aligned}
& J J \approx \int_{2 r}^{\infty} \int_{2 r \leq\left|z-x_{0}\right|<t}\left|b(z)-b_{B, w}\right|^{k} \frac{|f(z)| d z d t}{t^{n+1}} \\
& \leq \int_{2 r}^{\infty}\left(\int_{B\left(x_{0}, t\right)}\left|b(z)-b_{B, w}\right|^{k p^{\prime}} w(z)^{1-p^{\prime}} d z\right)^{1 / p^{\prime}} \\
& \times \frac{\|f\|_{L_{w}^{p}\left(B\left(x_{0}, t\right)\right)} d t}{t^{n+1}} \\
& \leq\|b\|_{*}^{k} \int_{2 r}^{\infty}\left(1+\ln \frac{t}{r}\right)^{k}\left\|w^{-1 / p}\right\|_{L^{p^{\prime}}\left(B\left(x_{0}, t\right)\right)} \\
& \quad \times \frac{\|f\|_{L_{w}^{p}\left(B\left(x_{0}, t\right)\right)} d t}{t^{n+1}} d t \\
& \leq[w]_{A_{p}}^{1 / p}\|b\|_{*}^{k} \int_{2 r}^{\infty} \ln ^{k}\left(e+\frac{t}{r}\right) \frac{\|f\|_{L_{w}^{p}\left(B\left(x_{0}, t\right)\right)}}{w\left(B\left(x_{0}, t\right)\right)^{1 / p}} \frac{d t}{t},
\end{aligned}
$$

where we use $w \in A_{p}$ in the last inequality. Combining the previous estimates for $J$ and $J$, we obtain

$$
\begin{aligned}
\left\|\left[b, G_{\alpha}\right]\left(f_{2}\right)\right\|_{L_{w}^{p}(B)} \leq & \|b\|_{*}^{k} w(B)^{1 / p} \\
& \times \int_{2 r}^{\infty} \ln ^{k}\left(e+\frac{t}{r}\right) \frac{\|f\|_{L_{w}^{p}\left(B\left(x_{0}, t\right)\right)}}{w\left(B\left(x_{0}, t\right)\right)^{1 / p}} \frac{d t}{t} .
\end{aligned}
$$

Therefore, it follows from the above estimates (62), (63), and (69) that

$$
\begin{aligned}
\left\|\left[b, G_{\alpha}\right](f)\right\|_{L_{w}^{p}(B)} \leq & \|b\|_{*}^{k} w(B)^{1 / p} \\
& \times \int_{2 r}^{\infty} \ln ^{k}\left(e+\frac{t}{r}\right) \frac{\|f\|_{L_{w}^{p}\left(B\left(x_{0}, t\right)\right)}}{w\left(B\left(x_{0}, t\right)\right)^{1 / p}} \frac{d t}{t} .
\end{aligned}
$$

By substitution of variables, we obtain

$$
\begin{aligned}
& \left\|\left[b, G_{\alpha}\right]^{k}(f)\right\|_{M^{p, q_{2}}(w)} \\
& \leq\|b\|_{*_{0} \in \sup _{\substack{0 \\
r>0}}^{k} \varphi_{2}\left(x_{0}, r\right)^{-1}} \\
& \quad \times \int_{2 r}^{\infty} \ln ^{k}\left(e+\frac{t}{r}\right) \frac{\|f\|_{L_{w}^{p}\left(B\left(x_{0}, t\right)\right)}}{w\left(B\left(x_{0}, t\right)\right)^{1 / p}} \frac{d t}{t} \\
& \leq\|b\|_{*}^{k} \sup _{x_{0} \in \mathbb{R}^{n}} \varphi_{2}\left(x_{0}, r^{-1}\right)^{-1} \\
& \quad \times \int_{0}^{r} \ln ^{k}\left(e+\frac{r}{s}\right) \frac{\|f\|_{L_{w}^{p}\left(B\left(x_{0}, 1 / s\right)\right)}}{w\left(B\left(x_{0}, 1 / s\right)\right)^{1 / p}} \frac{d s}{s} .
\end{aligned}
$$

Let $W(r)=\varphi_{2}\left(x_{0}, r^{-1}\right)^{-1} r$ and let $V(r)=\varphi_{1}\left(x_{0}, r^{-1}\right)^{-1}$ $w\left(B\left(x_{0}, r^{-1}\right)\right)^{-1 / p}$. Since $\left(\varphi_{1}, \varphi_{2}\right)$ satisfy condition (12), we can 
easily prove that $W(r)$ and $V(r)$ satisfy condition (32). Then, by Lemma 16, we obtain the following estimate:

$$
\left\|\left[b, G_{\alpha}\right]^{k}(f)\right\|_{M^{p, \varphi_{2}}(w)} \preceq\|b\|_{*}^{k}\|f\|_{M^{p, \varphi_{1}}(w)} .
$$

Proof of Theorem 9. We can prove it by using the the argument as in the proofs of Theorems 6 and 8. Here, the details are omitted.

Proof of Theorem $9^{\prime}$. The method of proof is the same as those of Theorems $6^{\prime}$ and 9. Here, we ignore its proof.

\section{Conflict of Interests}

The authors declare that they have no conflict of interests.

\section{References}

[1] L. Grafakos, Classical and Modern Fourier Analysis, Pearson Education, Upper Saddle River, NJ, USA, 2004.

[2] E. M. Stein, Singular Integrals and Differentiability Properties of Functions, Princeton University Press, Princeton, NJ, USA, 1970.

[3] E. M. Stein, Harmonic Analysis: Real-Variable Methods, Orthogonality, and Oscillatory Integrals, vol. 43 of Princeton Mathematical Series, Princeton University Press, Princeton, NJ, USA, 1993.

[4] M. Wilson, “The intrinsic square function," Revista Matemática Iberoamericana, vol. 23, no. 3, pp. 771-791, 2007.

[5] M. Wilson, Weighted Littlewood-Paley Theory and ExponentialSquare Integrability, vol. 1924 of Lecture Notes in Mathematics, Springer, Berlin, Germany, 2007.

[6] V. S. Guliyev and P. S. Shukurov, "Commutators of intrinsic square functions on generalized Morrey spaces," Proceedings of IMM of NAS of Azerbaijan, vol. 39, pp. 1-18, 2013.

[7] V. S. Guliyev, K. R. Rahimova, and M. N. Omarova, "Commutators of intrinsic square functions on vector-valued generalized Morrey spaces," Submitted.

[8] V. S. Guliyev and M. N. Omarova, "Commutators of intrinsic square Functions on vector-valued generalized weighted Morrey spaces," Submitted.

[9] V. S. Guliyev and M. N. Omarova, "Commutators of vectorvalued intrinsic square functions on vector-valued generalized weighted Morrey spaces," Submitted, http://arxiv.org/abs/ 1312.5587.

[10] J. Z. Huang and Y. Liu, "Some characterizations of weighted Hardy spaces," Journal of Mathematical Analysis and Applications, vol. 363, no. 1, pp. 121-127, 2010.

[11] A. K. Lerner, "Sharp weighted norm inequalities for LittlewoodPaley operators and singular integrals," Advances in Mathematics, vol. 226, no. 5, pp. 3912-3926, 2011.

[12] H. Wang, "Intrinsic square functions on the weighted Morrey spaces," Journal of Mathematical Analysis and Applications, vol. 396, no. 1, pp. 302-314, 2012.

[13] H. Wang, "Boundedness of intrinsic square functions on the weighted weak Hardy spaces," Integral Equations and Operator Theory, vol. 75, no. 1, pp. 135-149, 2013.

[14] H. Wang and H. Liu, "Weak type estimates of intrinsic square functions on the weighted Hardy spaces," Archiv der Mathematik, vol. 97, no. 1, pp. 49-59, 2011.
[15] H. Wang and H. P. Liu, "The intrinsic square function characterizations of weighted Hardy spaces," Illinois Journal of Mathematics, vol. 56, no. 2, pp. 367-381, 2012.

[16] H. Wang, "Weak type estimates for intrinsic square functions on weighted Morrey spaces," Analysis in Theory and Applications, vol. 29, no. 2, pp. 104-119, 2013.

[17] X. M. Wu and T. T. Zheng, "Commutators of intrinsic square functions on generalized Morrey spaces," Journal of Inequalities and Applications, vol. 2014, article 128, 2014.

[18] C. B. Morrey, "On the solutions of quasi-linear elliptic partial differential equations," Transactions of the American Mathematical Society, vol. 43, no. 1, pp. 126-166, 1938.

[19] T. Mizuhara, "Boundedness of some classical operators on generalized Morrey spaces," in ICM-90 Satellite Conference Proceedings: Harmonic Analysis, S. Igari, Ed., pp. 183-189, 1991.

[20] Y. Komori and S. Shirai, "Weighted Morrey spaces and a singular integral operator," Mathematische Nachrichten, vol. 282, no. 2, pp. 219-231, 2009.

[21] V. S. Guliyev, T. Karaman, and A. Serbetci, "Boundedness of sublinear operators generated by Calderón-Zygmund operators on generalized weighted Morrey Spaces," Annals of the Alexandru Ioan Cuza University - Mathematics, vol. 60, no. 1, pp. 227244, 2014.

[22] V. S. Guliyev, "Boundedness of classical operators and commutators of real analysis in generalized weighted Morrey spaces. Some applications," in Proceedings of the International Conference in Honour of Professor V.I. Burenkov on the Occasion of His 70th Birthday, vol. 20, Kirsehir, Turkey, May 2011.

[23] S. S. Aliev and V. S. Guliev, "Boundedness of the parametric Marcinkiewicz integral operator and its commutators on generalized Morrey spaces," Georgian Mathematical Journal, vol. 19, no. 2, pp. 195-208, 2012.

[24] A. Akbulut, V. S. Guliyev, and R. Mustafayev, "On the boundedness of the maximal operator and singular integral operators in generalized Morrey spaces," Mathematica Bohemica, vol. 137, no. 1, pp. 27-43, 2012.

[25] A. Eroglu, "Boundedness of fractional oscillatory integral operators and their commutators on generalized Morrey spaces," Boundary Value Problems, vol. 2013, article 70, 12 pages, 2013.

[26] Y. Fan, "Boundedness of sublinear operators and their commutators on generalized central Morrey spaces," Journal of Inequalities and Applications, vol. 2013, article 411, 2013.

[27] V. S. Guliyev, "Boundedness of the maximal, potential and singular operators in the generalized Morrey spaces," Journal of Inequalities and Applications, vol. 2009, Article ID 503948, 20 pages, 2009.

[28] V. S. Guliyev and L. G. Softova, "Global regularity in generalized Morrey spaces of solutions to nondivergence elliptic equations with VMO coefficients," Potential Analysis, vol. 38, no. 3, pp. 843-862, 2013.

[29] V. S. Guliyev, S. S. Aliyev, and T. Karaman, "Boundedness of a class of sublinear operators and their commutators on generalized Morrey spaces," Abstract and Applied Analysis, vol. 2011, Article ID 356041, 18 pages, 2011.

[30] V. S. Guliyev, "Generalized weighted Morrey spaces and higher order commutators of sublinear operators," Eurasian Mathematical Journal, vol. 3, no. 3, pp. 33-61, 2012.

[31] M. Carro, L. Pick, J. Soria, and V. D. Stepanov, "On embeddings between classical Lorentz spaces," Mathematical Inequalities and Applications, vol. 4, no. 3, pp. 397-428, 2001. 


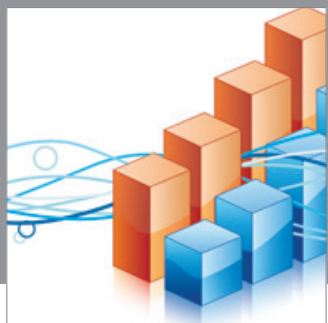

Advances in

Operations Research

mansans

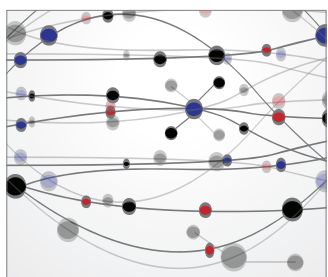

The Scientific World Journal
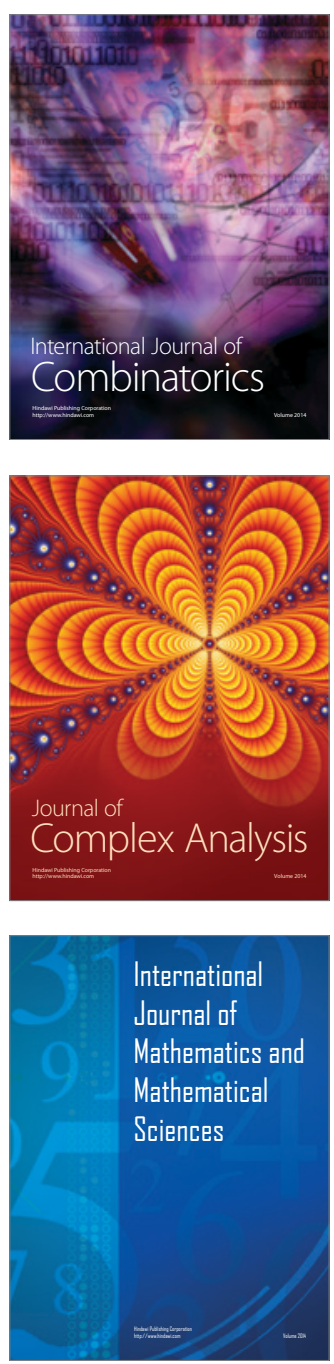
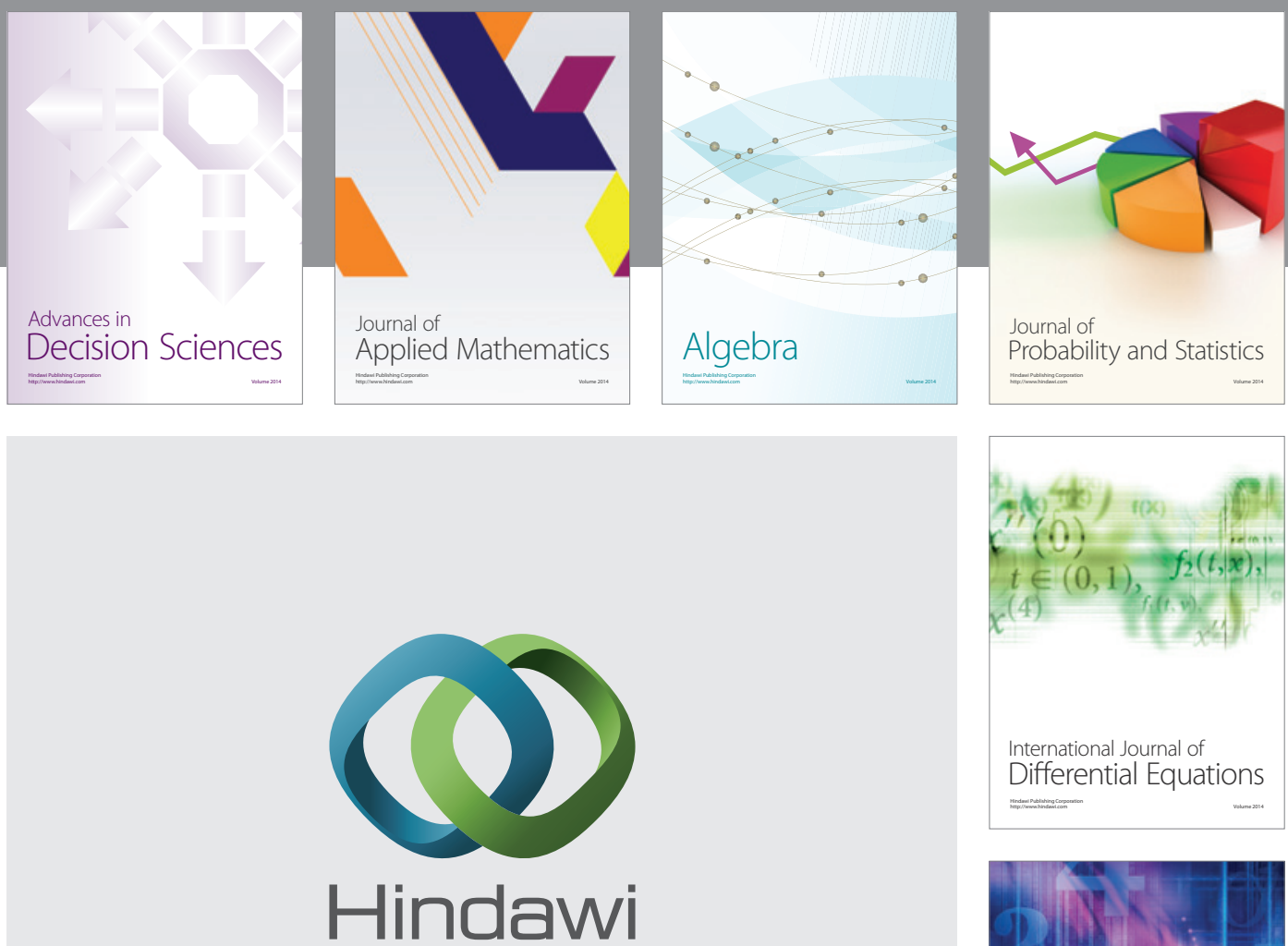

Submit your manuscripts at http://www.hindawi.com
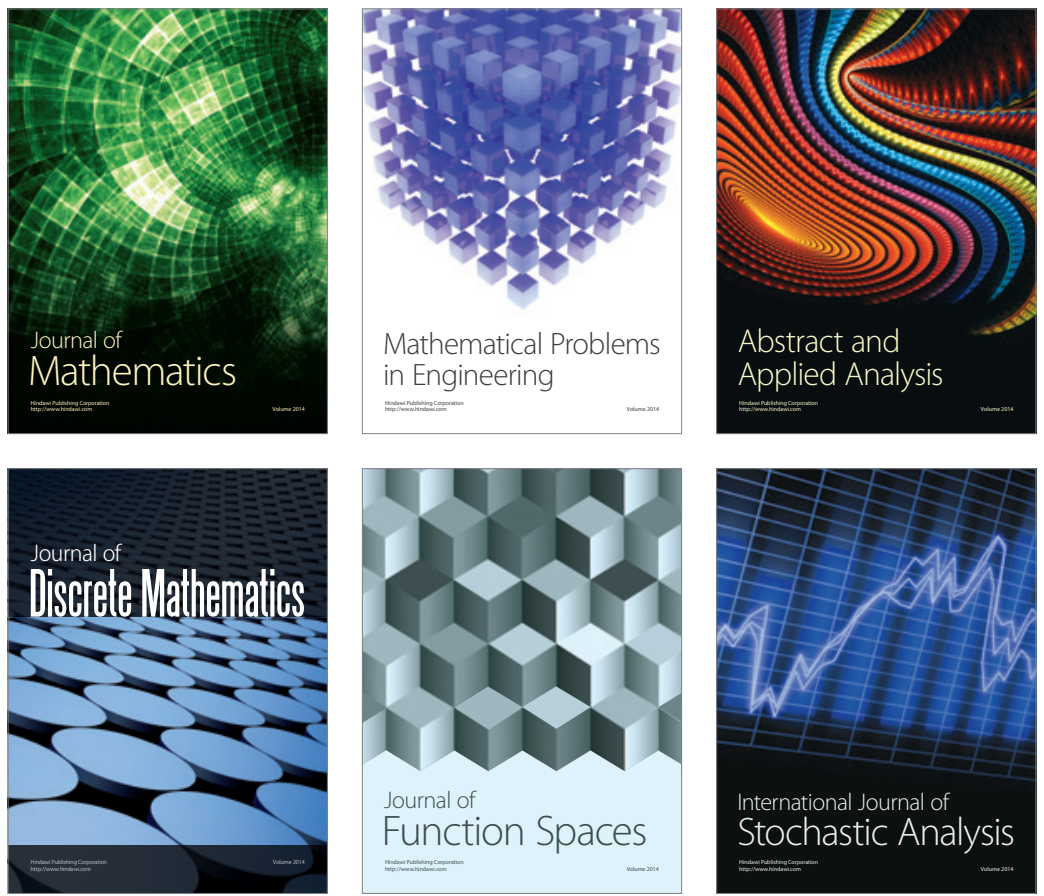

Journal of

Function Spaces

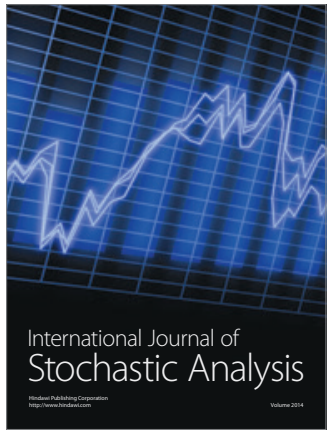

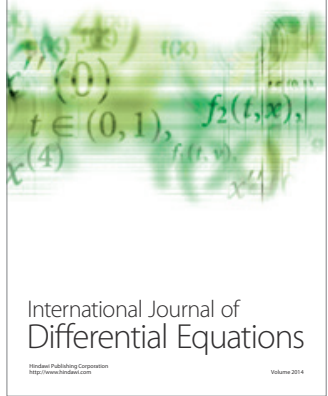
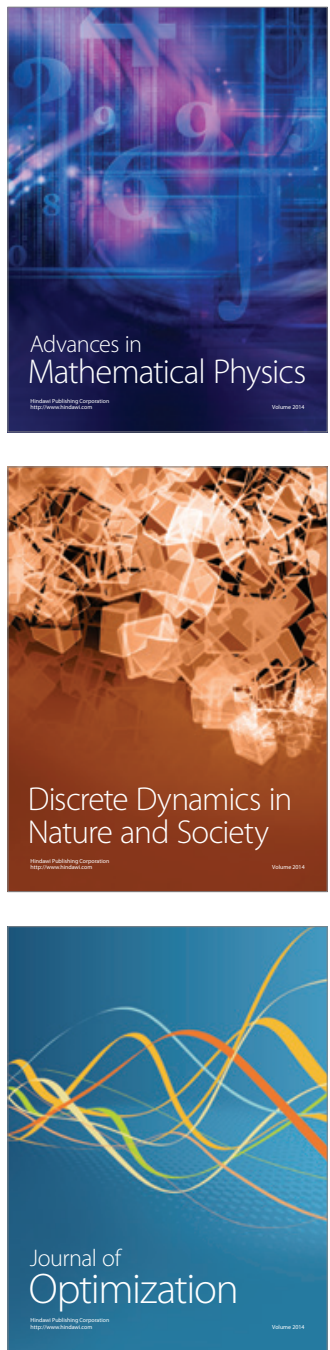